\title{
Radioembolisation with yttrium-90 microspheres versus sorafenib for treatment of advanced hepatocellular carcinoma (SARAH): study protocol for a randomised controlled trial
}

Valérie Vilgrain ${ }^{1,2^{*}}$, Mohamed Abdel-Rehim ${ }^{1}$, Annie Sibert ${ }^{1}$, Maxime Ronot ${ }^{1,2}$, Rachida Lebtahi ${ }^{3}$, Laurent Castéra ${ }^{4,5}$, Gilles Chatellier ${ }^{6,7,8}$ and on behalf of the SARAH trial group

\begin{abstract}
Background: Untreated advanced hepatocellular carcinoma (HCC) is linked to poor prognosis. While sorafenib is the current recommended treatment for advanced HCC, radioembolisation (RE; also called selective internal radiation therapy or SIRT) with yttrium-90 microspheres has shown efficacy in cohort studies. However, there are no head-to-head trials comparing radiation therapy with yttrium-90 microspheres and sorafenib in advanced HCC. The SARAH trial has been designed to compare the efficacy and safety of sorafenib therapy and RE using yttrium-90 resin microspheres (SIR-Spheres ${ }^{\mathrm{TM}}$; Sirtex Medical Limited, North Sydney, Australia) in patients with advanced HCC. Quality of life (QoL) and cost-effectiveness will also be compared between therapies.

Methods/Design: SARAH is a prospective, randomised, controlled, open-label, multicentre trial comparing the efficacy of RE with sorafenib in the treatment of patients with advanced HCC. The trial aims to recruit adults with a life expectancy of $>3$ months, Eastern Cooperative Oncology Group (ECOG) performance status $\leq 1$, and: advanced HCC according to the Barcelona criteria (stage C) or recurrent HCC after surgical or thermoablative treatment who are not eligible for surgical resection, liver transplantation or thermal ablation; or two rounds of failed chemoembolisation. Patients will be randomised 1:1 to receive either RE or sorafenib 400 mg twice daily. All patients will be monitored for between 12 and 48 months following start of treatment. The primary endpoint of the SARAH trial is overall survival (OS). Secondary endpoints include: adverse events, progression-free survival at 6 months; tumour response rate; general or liver disease-specific QoL scores; and cost of each treatment strategy. Assuming an increase in median OS of 4 months with RE versus sorafenib therapy, randomising at least 400 patients (200 in each treatment arm) will be sufficient for $80 \%$ power and a bilateral alpha risk of 5\%; therefore, 440 patients will be enrolled to allow for 10\% loss of patients due to ineligibility.
\end{abstract}

Discussion: The SARAH trial is the first randomised head-to-head study to compare RE with sorafenib in advanced HCC, and will establish the potential role of RE in HCC treatment guidelines.

Trial registration: ClinicalTrials.gov identifier NCT01482442, first received 28 November 2011

Keywords: Advanced hepatocellular carcinoma, Radioembolisation (RE), SIR-Spheres ${ }^{\mathrm{TM}}$ microspheres, Sorafenib

\footnotetext{
*Correspondence: valerie.vilgrain@bjn.aphp.fr

'Department of Radiology, Assistance Publique, Hôpitaux de Paris, APHP,

University Hospitals Paris Nord Val de Seine, Beaujon, 100 boulevard General Leclerc, 92118 Clichy, Hauts-de-Seine, France

University Paris Diderot, Sorbonne Paris Cité, Paris, France, INSERM U1149,

Centre de Recherche de I'Inflammation (CRI), 16 rue Henri Huchard, 75018

Paris, France

Full list of author information is available at the end of the article
} 


\section{Background}

The prevalence and incidence of hepatocellular carcinoma (HCC) is highly variable in different regions of the world but the burden is predicted to increase in the coming years [1]. In developed countries, early diagnosis of $\mathrm{HCC}$ is possible in 30 to $60 \%$ of patients, and as a result, $\mathrm{HCC}$ is often diagnosed in the advanced stage of disease (stage $\mathrm{C}$ of the Barcelona Clinic Liver Cancer classification - that is, ECOG performance status 1 to 2, portal invasion or extrahepatic spread, and Child-Pugh A-B). Curative treatment (by surgical resection, liver transplantation or thermoablative treatment) is possible only in a limited proportion of these patients [2], and many cases of HCC progress to an advanced stage following locoregional treatment. In patients with untreated advanced HCC, the prognosis is poor, with a median survival time of approximately 5 to 7 months, although this varies depending on Child-Pugh score [3-5].

The pivotal Sorafenib Hepatocellular carcinoma Assessment Randomized Protocol (SHARP) trial showed that sorafenib (Nexavar ${ }^{\mathrm{Tm}}$, Bayer HealthCare Pharmaceuticals, Berlin, Germany) treatment significantly increased median overall survival (OS) time by approximately 3 months versus placebo (10.7 months versus 7.9 months, respectively; $P<0.001$ ) in patients with advanced HCC [6]. These findings were subsequently confirmed in a randomised controlled trial in the Asia-Pacific, which showed OS of 6.5 months in the sorafenib arm versus 4.2 in the placebo arm $(P<0.014)$ [7]. As a result of these data, sorafenib is the current recommended first-line treatment for advanced (Barcelona stage C) HCC [2]. However, while sorafenib increased OS in the SHARP study, it did not improve median time to symptomatic progression, and was associated with an overall adverse-effect incidence of $80 \%$. Adverse events experienced by $>5 \%$ of patients in the SHARP trial included diarrhoea (13.1\%), asthenia (7.4\%), hand-foot skin reaction (7.0\%), and erythema or desquamation (5.4\%); dose reductions and treatment interruptions due to adverse effects occurred in $26 \%$ and $44 \%$ of cases, respectively [6]. As such, there is a medical need for the study of alternative treatment options for advanced HCC.

Radioembolisation (RE; also called selective internal radiation therapy or SIRT) with SIR-Spheres ${ }^{\mathrm{TM}}$ (Sirtex Medical Limited, North Sydney, Australia), which contain the $\beta$-emitter yttrium-90, is one potential alternative treatment of advanced HCC. RE enables targeted delivery of radiation to the tumours, while the surrounding liver parenchyma is largely spared. A recent meta-analysis showed a high response rate to yttrium-90 RE in HCC patients [8]. Population disparity prevented assessment of OS in this meta-analysis but cohort studies of patients with $\mathrm{HCC}$ receiving yttrium-90 RE report median OS between 7 and 26.3 months [9-18]. Collectively, these data suggest that the use of RE for advanced HCC warrants further investigation, and might improve median OS with fewer side effects and/or better quality of life (QoL) compared with sorafenib.

To the authors' knowledge, no controlled, prospective trials have been published on the efficacy of RE in HCC patients. For this reason, the SorAfenib versus Radioembolisation in Advanced Hepatocellular carcinoma (SARAH) trial has been designed as a prospective, randomised, open-label, multicentre trial to compare the OS in patients with advanced HCC receiving either RE with SIR-Spheres ${ }^{\mathrm{TM}}$ or sorafenib. Secondary objectives include comparisons between the treatment arms of other efficacy parameters, the safety profile and tolerability, QoL and cost-effectiveness.

\section{Methods/Design}

The SARAH trial will be conducted in accordance with the Declaration of Helsinki and current good clinical practice guidelines, and all participating centres have obtained the relevant ethics committee approval before patient enrolment (see Additional file 1).

\section{Eligible population}

The inclusion and exclusion criteria for the SARAH trial are summarised in Table 1 . Informed consent will be obtained from each participant.

\section{Overview of trial design}

SARAH is a prospective, randomised open-label, multicentre trial comparing RE and sorafenib in patients with advanced HCC. In SARAH, the aim will be to recruit a minimum of 440 patients over a period of 24 months across 28 centres in France. Centres will be chosen based on their potential to recruit a high number of patients, and the expertise in intra-arterial treatment, and will receive special training with RE. Eligible patients will be stratified 1:1 to receive either systemic therapy with oral sorafenib (control arm) or RE with SIR-Spheres ${ }^{\mathrm{Tm}}$ (RE arm; Figure 1).

\section{Randomisation}

Eligible patients will be randomised 1:1. The randomisation will be stratified by centre, Eastern Cooperative Oncology Group (ECOG) score (0 versus 1 ), the presence or absence of macroscopic vascular invasion seen on imaging (obstruction of the portal vein or its branches) and previous chemoembolisation failure. The list will be balanced by different sized blocks and randomly alternated. The data coordination centre will prepare the randomisation list before enrolment begins.

\section{Treatments}

In the sorafenib arm, patients will receive oral treatment with sorafenib (400 mg twice daily) beginning in the week following randomisation (the first day of receiving 
Table 1 Patient eligibility criteria for SARAH trial

Inclusion criteria
- Written informed consent provided
- Aged $\geq 18$ years
- Histologically or cytologically confirmed diagnosis, or AASLD criteria for
the diagnosis, of HCC and at least one measureable lesion on CT
according to RECIST criteria
- Patients not eligible for surgical resection, liver transplantation or
thermoablation who have advanced HCC according to the Barcelona criteria
(stage C), with or without portal invasion OR patients with recurrent HCC
(new lesion in a different place) after surgical or thermoablative treatment
who are not eligible for any other treatment; OR patients in whom
chemoembolisation has failed after two rounds - treatment failure is
defined as the absence of objective response in the treated nodule after
two rounds (objective response according to the modified RECIST
criteria and/or EASL criteria)

- ECOG performance status $\leq 1$

- Adequate haematological function: haemoglobin $\geq 9 \mathrm{~g} / 100 \mathrm{~mL}$, neutrophils $\geq 1,500 / \mathrm{mm}^{3}$, platelets $\geq 50,000 / \mathrm{mm}^{3}$

- Adequate kidney function: creatinine $<150 \mu \mathrm{mol} / \mathrm{L}$

- Bilirubin $\leq 50 \mu \mathrm{mol} / \mathrm{l}, \mathrm{AST}$ or $\mathrm{ALT} \leq 5 \times \mathrm{ULN}, \mathrm{INR} \leq 1.5$

- If liver cirrhosis, Child-Pugh A-B7

- Affiliated to a social security scheme or beneficiary

"Patients who have not responded to chemoembolisation but who meet the other selection criteria will be included in this study. AASLD, American Association for the Study of Liver Diseases; ALT, alanine aminotransferase; AST, aspartate transaminase; CT, computed tomography; EASL, European Association for the Study of the Liver; ECOG, Eastern Cooperative Oncology Group; HCC, hepatocellular carcinoma; INR, international normalised ratio; RECIST, response evaluation criteria in solid tumours; ULN, upper limit of normal.

treatment is defined as D0 in Table 2). Treatment suspensions and dose reductions (to $400 \mathrm{mg} / \mathrm{d}$ ) will be permitted in case of adverse events (based on the SHARP study [6]) and at the treating practitioner's discretion. Treatment may be resumed once the adverse events have been resolved, with incremental doses up to $400 \mathrm{mg}$ twice daily.

In the RE arm, patients randomised to RE will require a hepatic angiogram, and a liver-to-lung shunt pre-assessment with technetium-99 m ( $\left.{ }^{99 \mathrm{~m}} \mathrm{Tc}\right)$-marked human serum albumin in order to determine their suitability for the RE procedure. Accessory tumoural vascular branches and extrahepatic vascular branches will be embolised using thrombogenic coils or vascular plugs in order to isolate the arterial supply of the afferent vessel that vascularises

\section{Exclusion criteria}

- Other primary tumour except for basal cell carcinomas or superficial bladder cancers

- Extrahepatic metastases except non-specific pulmonary tumours $<1 \mathrm{~cm}$ and abdominal lymph nodes $<2 \mathrm{~cm}$

- Previously treated advanced HCC (excluding chemoembolisation ${ }^{*}$ )

- Advanced liver disease with a Child-Pugh score > B7 or active digestive haemorrhage or encephalopathy or refractory ascites

- Pregnant or breastfeeding women

- Allergy to contrast agents

- Contraindication to hepatic artery catheterisation, such as severe peripheral arterial disease precluding catheterisation

- Mental illness or other psychological disorder affecting the informed consent

- Patient unable or unwilling to comply with the treatment and follow-up required by the study

- Unable to take oral medication the tumour. A catheter will then be placed in the afferent vessel that vascularises the tumour, and 150 to $180 \mathrm{MBq}$ of ${ }^{99 \mathrm{~m}}$ Tc-marked human serum albumin will be injected. The injection rate and catheter position will mimic the anticipated RE procedure. After the injection, the patient will have the pulmonary shunt evaluated using a dual-head gamma camera or single-photon emission computed tomography (SPECT)/computed tomography (CT). The prescribed activity of SIR-Spheres ${ }^{\text {tux }}$ will then be calculated based on the patient's body surface area and the percentage tumour involvement as described by Kennedy et al. [19]. Patients who would receive a dose in the lungs higher than 25 Gy based on the liver-to-lung shunt preassessment will not receive RE, but will remain in this

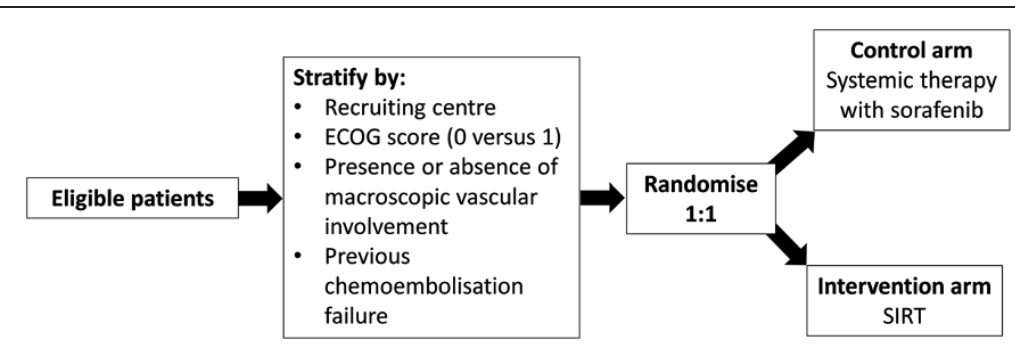

Figure 1 Overview of the SARAH trial design. ECOG, Eastern Cooperative Oncology Group; RE, radioembolisation. 
Table 2 SARAH trial assessment schedule

\begin{tabular}{|c|c|c|c|c|c|c|c|c|c|c|c|c|c|}
\hline Visits & Enrolment/randomisation & Do & D15 & M1 & M2 & M3 & M4 & M5 & M6 & M7 & M8 & M9 & End of participation \\
\hline Identification & $x$ & & & & & & & & & & & & \\
\hline Verification of selection criteria & $x$ & & & & & & & & & & & & \\
\hline Consent signature & $x$ & & & & & & & & & & & & \\
\hline Initial assessment - history & $x$ & & & & & & & & & & & & \\
\hline CT scan & $x$ & & & $x$ & & $x$ & & & $x$ & & & $x$ & $x$ \\
\hline CT perfusion & $x$ & & & $x$ & & $x$ & & & $x$ & & & & \\
\hline Laboratory tests & $x$ & $x$ & $x$ & $x$ & $x$ & $x$ & $x$ & $x$ & $x$ & $x$ & $x$ & $x$ & $x$ \\
\hline Classification & $x$ & & & $x$ & $x$ & $x$ & $x$ & $x$ & $x$ & $x$ & $x$ & $x$ & $x$ \\
\hline Clinical examination & & & & $x$ & $x$ & $x$ & $x$ & $x$ & $x$ & $x$ & $x$ & $x$ & $x$ \\
\hline Quality of life questionnaires & $x$ & & & $x$ & & $x$ & & & $x$ & & & $x$ & $x$ \\
\hline Preparatory angiography & & $x$ & & & & & & & & & & & \\
\hline Scintigraphy & & $x$ & & & & & & & & & & & \\
\hline RE & & $x$ & & & & & & & & & & & \\
\hline Start of sorafenib treatment & & $x$ & & & & & & & & & & & \\
\hline Retreatment $^{*}$ & & & & $x$ & $x$ & $x$ & $x$ & $x$ & $x$ & $x$ & $x$ & $x$ & \\
\hline Cancer progression monitoring & & & & $x$ & $x$ & $x$ & $x$ & $x$ & $x$ & $x$ & $x$ & $x$ & $x$ \\
\hline Sorafenib monitoring & & & & $x$ & $x$ & $x$ & $x$ & $x$ & $x$ & $x$ & $x$ & $x$ & $x$ \\
\hline Concomitant medication & & & & $x$ & $x$ & $x$ & $x$ & $x$ & $x$ & $x$ & $x$ & $x$ & $x$ \\
\hline Adverse events & & & & $x$ & $x$ & $x$ & $x$ & $x$ & $x$ & $x$ & $x$ & $x$ & $x$ \\
\hline
\end{tabular}

"Timing of retreatment depends upon type of retreatment (see text). CT, computed tomography; $D$, day; M, month; RE, radioembolisation.

study arm as part of the intention-to-treat group (ITT). Patients who are eligible for RE will commence treatment between the second and fifth week after randomisation with a single session of treatment (the day on which RE is administered is defined as D0 in Table 2). In patients with bilobar involvement, contralateral RE will be administered within 30 to 60 days. In order to avoid premature retreatment with RE of any lobe due to late tumour response, retreatment with RE will only be considered beyond 3 months from D0 in the absence of objective response or if there is significant progression on imaging (stable or progressor according to the response evaluation criteria in solid tumours (RECIST) or European Association for the Study of the Liver (EASL)) in the treated region (same tumour or new tumour). Retreatment with RE will also be considered beyond 6 weeks in the event of partial failure of the initial treatment due to an identified correctible cause or if an insufficient tumour dose was delivered.

\section{Trial assessments}

The last enrolled patient will be followed up for 12 months after the start of treatment (D0). All other patients will be followed up until the final visit of the last enrolled patient. Patients will therefore be followed up for a maximum of 48 months and a minimum of 12 months following start of treatment. All patients will be assessed by the schedule summarised in Table 2. Treatment will be discontinued if the patient withdraws consent, if the treating physician deems it necessary for medical reasons or if a serious adverse event occurs - after discontinuation, patients should be assessed by $\mathrm{CT}$ as soon as possible to assess response to treatment.

\section{Outcome measures}

The primary endpoint of the SARAH trial is OS. Secondary endpoints include: adverse events rate, progression-free survival (PFS) at 6 months according to RECIST [20], modified RECIST, EASL and Choi criteria; tumour response rate; general or liver disease-specific QoL scores; and cost of each strategy.

Cost endpoints are: the cost of RE from the hospital perspective; the average cost per patient from the payer's perspective; and the incremental cost-effectiveness or cost-utility ratio.

\section{Outcome definitions}

- OS is defined as the time from the date of randomisation to death from any cause.

- PFS - the time from the date of first treatment to disease progression - and tumour response rate (complete response, partial response, stability, or progression) will be determined from serial CT scans using RECIST, modified RECIST, EASL criteria for $\mathrm{HCC}$, and Choi criteria [21]. Radiological examinations will be conducted by abdominal radiologists at each 
centre followed by a separate centralised review of radiological examinations.

- The general and liver disease-specific quality of life scores will be calculated using the European Organisation for Research and Treatment of Cancer (EORTC) quality of life questionnaire (QLQ-C30) version 3 and the HCC-specific QLQ-HCC 18 questionnaire [22].

- Adverse events will be reported according to National Cancer Institute criteria (National Cancer Institute Common Terminology criteria for Adverse Events (NCI CTCAE) Version 4.0) [23].

The cost of therapy from the hospital's perspective will be estimated by including all the resources that are directly attributable to the procedure (that is, equipment, tests, total work time, and so on), which will be assigned a value based on the purchase price by the hospital. The mean overall cost per patient from the payer's perspective will include the relative stay index and readmissions during the patient's follow-up period. Calculation of the incremental cost-effectiveness ratio per year of survival or the incremental cost-utility ratio between RE and sorafenib will be complemented by the bootstrap resampling method and an acceptability curve for the cost-effectiveness ratio.

\section{Sample size calculation and statistical considerations}

Based on OS data with sorafenib from the SHARP study [6] and with yttrium-90 RE reported in the literature [9,11-15], the number of patients required for randomisation to detect a clinically relevant increase (4 months) in OS time with RE versus sorafenib was determined as 400 patients (200 patients in each treatment arm). This translates to an expected median OS time of 10.7 months in the sorafenib group and 15 months in the RE group, with an accrual period of 24 months and follow-up of 12 months. These guarantee a power of $80 \%$ with a bilateral alpha risk of $5 \%$. Estimating that up to $10 \%$ of patients that are recruited will not fulfil the criteria of eligibility for randomisation, we aimed to enrol 440 patients.

A Data Monitoring Committee (DMC) will regularly review the toxicity data to assess the safety profile of the treatment (including serious adverse events and mortality). The first intermediate analysis will take place once 30 patients have been followed up for at least 2 months in each treatment arm, after which the DMC will convene every 6 months.

\section{Statistical analysis}

Results will be reported according to the Consolidated Standards of Reporting Trials (CONSORT) statement. An ITT analysis will be performed, keeping patients in their randomisation group and including protocol deviations. A 'per-protocol' sensitivity analysis will also be performed. A study flowchart will be provided, including the number of patients who: are eligible; are randomised to receive treatment; are followed up; withdraw from the study; and are lost to follow-up. Major protocol deviations and the reasons for withdrawal from the study will be described.

The Kaplan and Meier method will be used to calculate survival (OS and PFS). The comparison of survival rates at 12 months between the two treatment groups will be performed using the log rank test (Mantel-Haenszel version). In addition, the treatment effect, once adjusted for the stratified randomisation factors, will be calculated via: (1) a stratified log rank analysis; and (2) a Cox's regression model.

The median survival times (OS and PFS) in both treatment groups will be calculated, along with the confidence interval associated with the difference or with the median survival time ratio [24].

Toxicity will be reported according to NCI CTCAE Version 3.0, with particular reference to the proportion of patients experiencing grade $3 / 4$ toxicity in each treatment arm.

The objective response will be determined via the RECIST and modified ( $\mathrm{m}$ )RECIST criteria, the EASL criteria for HCC, and the Choi criteria, and a comparison will be made between the two treatment groups using the 'best response during follow-up' criterion. The response rates will be calculated by comparing the number of patients who responded during follow-up (complete or partial response) with the total number of randomised patients in each group. The related confidence intervals will be calculated and compared between the two groups using Pearson's chi-squared test.

\section{Economic evaluation and statistical methods}

The costs of both therapies will be compared using the Student's $t$ test. Uncertainty over the cost and effectiveness differentials between the two groups will be measured using the bootstrap resampling method. Cost-effectiveness will be measured using the incremental cost-effectiveness ratio per life year gained. Markov modelling will be used to calculate the cost-effectiveness ratio by simulating patient follow-up beyond the end of the study.

\section{Discussion}

The SARAH trial will compare the efficacy and safety of $\mathrm{RE}$ with that of sorafenib in the treatment of advanced HCC (see Additional file 2). To the authors' knowledge, no prospective, controlled trials have been published, randomised or otherwise, on the efficacy of yttrium-90 RE in patients with HCC.

Sorafenib has been chosen as the control in the SARAH trial as it is the current recommended first-line treatment 
for advanced (Barcelona stage C) HCC $[2,6,25]$. Median OS is significantly increased by nearly 3 months with sorafenib versus placebo [6,7]. However, the incidence of adverse events was high $(80 \%)$ in the SHARP trial and there was no improvement in time to symptomatic progression with sorafenib therapy [6]. Attempts to improve outcomes for patients with advanced HCC on sorafenib by combination with other drug therapies have had limited success when compared with sorafenib alone [26-30]. Sorafenib combination therapy with transarterial chemoembolisation (TACE) has shown promise in patients with unresectable HCC [31], but is not currently recommended for advanced HCC. Indeed, combination therapy with sorafenib is not currently recommended outside the clinical trial setting for advanced HCC [27], and there is a need for an efficacious alternative with a favourable safety profile. RE with yttrium-90 is also indicated as a first-line treatment for unresectable HCC in a number of countries, and is associated with a high response rate in HCC patients (78 to $89 \%$ across 14 studies) [8]. Thus a head-tohead, prospective trial of these two treatments warrants investigation.

Some aspects of the SARAH study design are worth further discussion. The inclusion criteria are similar to the SHARP trial, except extrahepatic dissemination is not permitted in the SARAH study as RE is a localised therapy. Although previous treatment for advanced HCC is an exclusion criterion, prior chemoembolisation is permitted as patients failing chemoembolisation would be indicated for sorafenib therapy. Eligible patients in the SARAH study have also been stratified according to ECOG score, as this is an independent prognostic factor for survival in patients with HCC treated with RE or sorafenib $[10,32]$. In addition, patients will be stratified by presence or absence of macroscopic vascular involvement as vascular involvement has been associated with poor prognosis in patients with HCC $[10,13,33]$.

In the SARAH trial, OS has been chosen as the primary endpoint as it is a more robust measure than PFS, and the SHARP trial demonstrated the efficacy of sorafenib based on this criterion. Moreover, the kinetics of tumour progression, as assessed from imaging techniques, is different between RE and sorafenib, rendering OS the best option for comparison between arms in this trial. An Asian study with similar inclusion criteria has now commenced, which compares sorafenib with RE in patients with locally advanced HCC [34], and could be used for meta-analysis in the future.

In addition to efficacy analyses, the SARAH trial offers the possibility of rigorously confirming the toxicity caused by sorafenib versus RE in patients with advanced HCC. This is important as the cardiovascular toxicity of sorafenib has been highlighted in a meta-analysis of cancer patients (predominantly renal carcinoma) [35], but was not a common complication in the SHARP study [6]. The SARAH trial will also offer the opportunity to conduct ancillary studies (for example, dosimetry and CT perfusion), and compare the cost of each treatment. A definitive economic evaluation of sorafenib therapy versus RE for advanced HCC is not currently available. It is therefore useful to: conduct a study using national data to establish the value of the resources used; take the specificities of local oncology practices into account; and compare sorafenib treatment to an up-to-date therapy, RE.

Limitations to the SARAH study design have been combated where feasible. While blinding is not possible due to the treatment methods, the potential biases caused by the lack of blinding have been minimised by the choice of OS as a robust primary endpoint. In addition, it is planned that an independent group of radiologists will perform a blind review of the imaging in order to guarantee the absence of bias regarding PFS.

The results from the SARAH trial should further the understanding of RE and determine the optimal treatment modality in advanced HCC. In addition, the data generated from this study may help to place RE into future consensus guidelines.

\section{Trial status}

The SARAH trial is currently recruiting participants.

\section{Additional files}

Additional file 1: Approval of ethics committee for all participating centres.

Additional file 2: Brief summary.

\begin{abstract}
Abbreviations
${ }^{99 m}$ Tc: technetium-99 m; AASLD: American Association for the Study of Liver Diseases; ALT: alanine aminotransferase; AST: aspartate transaminase; CONSORT: Consolidated Standards of Reporting Trials; CT: computed tomography; DMC: Data Monitoring Committee; EASL: European Association for the Study of the Liver; ECOG: Eastern Cooperative Oncology Group; EORTC: European Organisation for Research and Treatment of Cancer; HCC: hepatocellular carcinoma; INR: international normalised ratio; ITT: intention to treat; NCI CTCAE: National Cancer Institute Common Terminology Criteria for Adverse Events; OS: overall survival; PFS: progression-free survival; QoL: quality of life; RE: radioembolisation; RECIST: response evaluation criteria in solid tumours; SARAH: SorAfenib versus Radioembolisation in Advanced Hepatocellular carcinoma; SHARP: Sorafenib Hepatocellular carcinoma Assessment Randomized Protocol; SIRT: selective internal radiation therapy; SPECT: single-photon emission computed tomography; TACE: transarterial chemoembolisation; ULN: upper limit of normal.
\end{abstract}

\section{Competing interests}

W has received speaker fees from Sirtex. MAR, AS, MR, RL, LC and GC have no conflicts of interests.

\section{Authors' contributions}

W is Coordinating Investigator for the SARAH trial, made substantial contributions to the conception and design of the SARAH trial, drafted the manuscript, and revised the manuscript critically for important intellectual content. MAR made substantial contributions to the conception 
and design of the SARAH trial, drafted the manuscript, and revised the manuscript critically for important intellectual content. AS made substantial contributions to the conception and design of the SARAH trial, drafted the manuscript, and revised the manuscript critically for important intellectual content. MR made substantial contributions to the conception and design of the SARAH trial, drafted the manuscript, and revised the manuscript critically for important intellectual content. RL is a Principal Investigator for the SARAH trial, drafted the manuscript, and revised the manuscript critically for important intellectual content. LC is a Principal Investigator for the SARAH trial, made substantial contributions to the conception and design of the SARAH trial, drafted the manuscript, and revised the manuscript critically for important intellectual content. GC made substantial contributions to the conception and design of the SARAH trial, drafted the manuscript, and revised the manuscript critically for important intellectual content. All authors read and approved the final manuscript and agree to be accountable for all aspects of the work in ensuring that questions related to the accuracy or integrityof any part of the work are appropriately investigated and resolved.

\section{Acknowledgements}

We thank all the investigators involved in the SARAH study: René Adam, Isabelle Archambeaud, Eric Assenat, Christophe Aubé, Philippe Bachellier, Gabriele Barabino, Hélène Barraud, Christophe Bazin, Nadia Bouarioua, Hatem Boulahdour, Isabelle Brenot-Rossi, Christophe Cassinotto, Maria-Angéla Castilla-Lievre, Julien Cazejust, Jean-Pierre Cercueil, Dominique Charneau, Patrick Chevallier, Charlotte Costentin, Sylvie Costo, Olivier Couturier, Muriel Cuilleron, Thông Dao, Thierry de Baere, Eric Delabrousse, Elisabeth Delhom-Christol, Jean Yves Devaux, Vincent di Martino, Bernard Duclos, Michel Ducreux, Jérôme Dumortier, Benoît Dupas, Julien Edeline, Thomas Eugene, Benoît Gallix, Etienne Garin, René Gerolami, Francesco Giammarile, Michel Greget, Gilles Grimon, Martine Guyot, Francois Habersetzer, Alexandria Heurgue-Berlot, Patrick Hillon, Alina Diana Ilonca, Alessio Imperiale, Emmanuel Itti, Vincent Leroy, Maïté Lewin, Alain Luciani, Jean Lumbroso, Abakar Mahamat, Sophie Maitre, Claude Marcus, Lysiane Marthey, Elodie Mathias, Philippe Merle, Julie Morvan, Olivier Mundler, Eric Nguyen-Khac, Frédéric Oberti, Georges-Philippe Pageaux, Jean Pierre Pelage, Rémi Perdrisot, Nathalie Prevot-Bitot, Jean Luc Raoul, Micheline Razzouk, Agnès Rode, Yan Rolland, Olivier Rosmorduc, Julie Roux, Didier Samuel, Marc Sapoval, Anthony Sarran, Jean-Francois Seitz, Christian Sengel, Olivier Seror, Christine Silvain, Daniele Sommacale, Julien Taieb, Jean Pierre Tasu, Michel Toubeau, Jean-Claude Trinchet, Pierre Jean Valette, Julien Vergniol, Vincent Vidal, Julie Vincent, Pierre Weinmann, and Thierry Yzet. This study is sponsored by Assistance Publique-Hôpitaux de Paris, with financial assistance provided by Sirtex Technology Ltd. The authors acknowledge the editorial assistance provided by Martin Gilmour and Daniella Otway of ESP Bioscience (Crowthorne, UK) funded by Sirtex, during the preparation of this manuscript. The authors would like to thank Dr Juliette Djadi-Prat and Pascaline Aucouturier for their help in managing the SARAH study.

\section{Author details}

${ }^{1}$ Department of Radiology, Assistance Publique, Hôpitaux de Paris, APHP, University Hospitals Paris Nord Val de Seine, Beaujon, 100 boulevard General Leclerc, 92118 Clichy, Hauts-de-Seine, France. University Paris Diderot, Sorbonne Paris Cité, Paris, France, INSERM U1149, Centre de Recherche de I'Inflammation (CRI), 16 rue Henri Huchard, 75018 Paris, France. ${ }^{3}$ Department of Nuclear Medicine, Assistance Publique, Hôpitaux de Paris, APHP, University Hospitals Paris Nord Val de Seine, Beaujon, 100 boulevard General Leclerc, 92118 Clichy, Hauts-de-Seine, France. ${ }^{4}$ Department of Hepatology, Assistance Publique, Hôpitaux de Paris, APHP, University Hospitals Paris Nord Val de Seine, Beaujon, 100 boulevard General Leclerc, 92118 Clichy, Hauts-de-Seine, France. ${ }^{5}$ University Paris Diderot, Sorbonne Paris Cité, 5 rue Thomas Mann, 75013 Paris, France. ${ }^{6}$ Assistance Publique, Hôpitaux de Paris, APHP, Hôpital Européen Georges Pompidou, URC, 20 rue Leblanc, 75015 Paris, France. ${ }^{7}$ INSERM, Centre d'Investigation Épidémiologique 4 (CIE4), 80 rue Lecourbe, 75015 Paris, France. ${ }^{8}$ Université Paris-Descartes, Sorbonne Paris Cité, Faculté de Médecine, 12 rue de l'Ecole de Médecine, 75006 Paris, France.

Received: 22 July 2014 Accepted: 6 November 2014

Published: 3 December 2014

\section{References}

1. Venook AP, Papandreou C, Furuse J, de Guevara LL: The incidence and epidemiology of hepatocellular carcinoma: a global and regional perspective. Oncologist 2010, Suppl 4:5-13.

2. European Association for the Study of the Liver, European Organisation for Research and Treatment of Cancer: EASL-EORTC clinical practice guidelines: management of hepatocellular carcinoma. J Hepatol 2012, 56:908-943.

3. Cabbibo G, Enea M, Latteri F, Genco C, Craxì A, Cammá C: Survival of unresectable hepatocellular carcinoma: a meta-analysis of the control arms of 28 randomized trials. J Hepatol 2009, 50:S285.

4. Hsu C, Shen YC, Cheng CC, Hu FC, Cheng AL: Geographic difference in survival outcome for advanced hepatocellular carcinoma: implications on future clinical trial design. Contemporary Clinical Trial 2010, 31:55-61.

5. Llovet JM, Di Bisceglie AM, Bruix J, Kramer BS, Lencioni R, Zhu AX, Sherman M, Schwartz M, Lotze M, Talwalkar J, Gores GJ: Design and endpoints of clinical trials in hepatocellular carcinoma. J Natl Cancer Inst 2008, 100:698-711.

6. Llovet JM, Ricci S, Mazzaferro V, Hilgard P, Gane E, Blanc JF, de Oliveira AC, Santoro A, Raoul JL, Forner A, Schwartz M, Porta C, Zeuzem S, Bolondi L, Greten TF, Galle PR, Seitz JF, Borbath I, Häussinger D, Giannaris T, Shan M, Moscovici M, Voliotis D, Bruix J, SHARP Investigators Study Group: Sorafenib in advanced hepatocellular carcinoma. N Engl J Med 2008, 359:378-390.

7. Cheng AL, Kang YK, Chen Z, Tsao CJ, Qin S, Kim JS, Luo R, Feng J, Ye S, Yang TS, Xu J, Sun Y, Liang H, Liu J, Wang J, Tak WY, Pan H, Burock K, Zou J, Voliotis D, Guan Z: Efficacy and safety of sorafenib in patients in the Asia-Pacific region with advanced hepatocellular carcinoma: a phase III randomised, double-blind, placebo-controlled trial. Lancet Oncol 2009, 10:25-34.

8. Vente MA, Wondergem M, van der Tweel I, van den Bosch MA, Zonnenberg BA, Lam MG, van Het Schip AD, Nijsen JF: Yttrium-90 microsphere radioembolization for the treatment of liver malignancies: a structured meta-analysis. Eur Radiol 2009, 19:951-959.

9. Kulik LM, Atassi B, van Holsbeeck L, Souman T, Lewandowski RJ, Mulcahy MF, Hunter RD, Nemcek AA Jr, Abecassis MM, Haines KG 3rd, Salem R: Yttrium-90 microspheres (TheraSphere) treatment of unresectable hepatocellular carcinoma: downstaging to resection, RFA and bridge to transplantation. J Surg Oncol 2006, 94:572-586.

10. Sangro B, Carpanese L, Cianni R, Golfieri R, Gasparini D, Ezziddin S, Paprottka PM, Fiore F, Van Buskirk M, Bilbao Jl, Ettorre GM, Salvatori R, Giampalma E, Geatti O, Wilhelm K, Hoffmann RT, Izzo F, Iñarrairaegui M, Maini CL, Urigo C, Cappelli A, Vit A, Ahmadzadehfar H, Jakobs TF, Lastoria S, European Network on Radioembolization with Yttrium-90 Resin Microspheres (ENRY): Survival after yttrium-90 resin microsphere radioembolization of hepatocellular carcinoma across Barcelona clinic liver cancer stages: a European evaluation. Hepatology (Baltimore, MD) 2011, 54:868-878.

11. Lau WY, Ho S, Leung TW, Chan M, Ho R, Johnson PJ, Li AK: Selective internal radiation therapy for nonresectable hepatocellular carcinoma with intraarterial infusion of 90yttrium microspheres. Int J Radiat Oncol Biol Phys 1998, 40:583-592.

12. Lau WY, Leung WT, Ho S, Leung NW, Chan M, Lin J, Metreweli C, Johnson P, Li AK: Treatment of inoperable hepatocellular carcinoma with intrahepatic arterial yttrium-90 microspheres: a phase I and II study. Br J Cancer 1994, 70:994-999.

13. Kulik LM, Carr BI, Mulcahy MF, Lewandowski RJ, Atassi B, Ryu RK, Sato KT, Benson A 3rd, Nemcek AA Jr, Gates VL: Safety and efficacy of $90 \mathrm{Y}$ radiotherapy for hepatocellular carcinoma with and without portal vein thrombosis. Hepatology (Baltimore, MD) 2008, 47:71-81.

14. Sangro B, Bilbao Jl, Boan J, Martinez-Cuesta A, Benito A, Rodriguez J, Panizo A Gil B, Inarrairaegui M, Herrero I, Quiroga J, Prieto J: Radioembolization using 90Y-resin microspheres for patients with advanced hepatocellular carcinoma. Int J Radiat Oncol Biol Phys 2006, 66:792-800.

15. Young JY, Rhee TK, Atassi B, Gates VL, Kulik L, Mulcahy MF, Larson AC, Ryu RK, Sato KT, Lewandowski RJ, Omary RA, Salem R: Radiation dose limits and liver toxicities resulting from multiple yttrium-90 radioembolization treatments for hepatocellular carcinoma. J Vasc Intervent Radiol 2007, 18:1375-1382

16. Hilgard P, Hamami M, El Fouly A, Scherag A, Müller S, Ertle J, Heusner T, Cicinnati VR, Paul A, Bockisch A, Gerken G, Antoch G: Radioembolization with yttrium-90 glass microspheres in hepatocellular carcinoma: European Experience on safety and long-term survival. Hepatology 2010, 52:1741-1749. 
17. Mazzaferro V, Sposito C, Bhoori S, Romito R, Chiesa C, Morosi C, Maccauro M, Marchianò A, Bongini M, Lanocita R, Civelli E, Bombardieri E, Camerini T, Spreafico C: Yttrium-90 radioembolization for intermediate-advanced hepatocellular carcinoma: a phase 2 study. Hepatology 2013, 57:1826-1837.

18. Salem R, Lewandowski RJ, Mulcahy MF, Riaz A, Ryu RK, Ibrahim S, Atassi B, Baker T, Gates V, Miller FH, Sato KT, Wang E, Gupta R, Benson AB, Newman SB, Omary RA, Abecassis M, Kulik L: Radioembolization for hepatocellular carcinoma using yttrium-90 microspheres: a comprehensive report of long-term outcomes. Gastroenterology 2010, 138:52-64.

19. Kennedy AS, Kleinstreuer C, Basciano CA, Dezarn WA: Computer modeling of yttrium-90-microsphere transport in the hepatic arterial tree to improve clinical outcomes. Int J Radiat Oncol Biol Phys 2010, 76:631-637.

20. Therasse P, Arbuck SG, Eisenhauer EA, Wanders J, Kaplan RS, Rubinstein L, Verweij J, Van Glabbeke M, van Oosterom AT, Christian MC, Gwyther SG: New guidelines to evaluate the response to treatment in solid tumors. European Organization for Research and Treatment of Cancer, National Cancer Institute of the United States, National Cancer Institute of Canada. J Natl Cancer Inst 2000, 92:205-216.

21. Ronot $M$, Bouattour $M$, Wassermann J, Bruno O, Dreyer C, Larroque $B$, Castera L, Vilgrain V, Belghiti J, Raymond E, Faivre S: Alternative Response Criteria (Choi, European association for the study of the liver, and modified Response Evaluation Criteria in Solid Tumors [RECIST]) Versus RECIST 1.1 in patients with advanced hepatocellular carcinoma treated with sorafenib. Oncologist 2014, 19:394-402.

22. Blazeby JM, Currie E, Zee BC, Chie WC, Poon RT, Garden OJ, Group EQoL: Development of a questionnaire module to supplement the EORTC QLQ-C30 to assess quality of life in patients with hepatocellular carcinoma, the EORTC QLQ-HCC18. Eur J Cancer 2004, 40:2439-2444.

23. Common Terminology Criteria for Adverse Events (CTCAE) Version 4.0. http://evs.nci.nih.gov/ftp1/CTCAE/CTCAE_4.03_2010-06-14_QuickReference_5x7.pdf]

24. Su JQ, Wei LJ: Nonparametric estimation for the difference or ratio of median failure times. Biometrics 1993, 49:603-607.

25. El-Serag HB, Marrero JA, Rudolph L, Reddy KR: Diagnosis and treatment of hepatocellular carcinoma. Gastroenterology 2008, 134:1752-1763.

26. Abou-Alfa GK, Johnson P, Knox JJ, Capanu M, Davidenko I, Lacava J, Leung T, Gansukh B, Saltz LB: Doxorubicin plus sorafenib vs doxorubicin alone in patients with advanced hepatocellular carcinoma: a randomized trial. JAMA 2010, 304:2154-2160.

27. Abdel-Rahman O, Fouad M: Sorafenib-based combination as a first line treatment for advanced hepatocellular carcinoma: a systematic review of the literature. Crit Rev Oncol/Hematol 2014, 91:1-8.

28. Gomez-Martin C, Bustamante J, Castroagudin JF, Salcedo M, Garralda E, Testillano M, Herrero I, Matilla A, Sangro B: Efficacy and safety of sorafenib in combination with mammalian target of rapamycin inhibitors for recurrent hepatocellular carcinoma after liver transplantation. Liver Transplant 2012, 18:45-52.

29. Petrini I, Lencioni M, Ricasoli M, lannopollo M, Orlandini C, Oliveri F, Bartolozzi C, Ricci S: Phase II trial of sorafenib in combination with 5-fluorouracil infusion in advanced hepatocellular carcinoma. Cancer Chemother Pharmacol 2012, 69:773-780.

30. Prete SD, Montella L, Caraglia M, Maiorino L, Cennamo G, Montesarchio V, Piai G, Febbraro A, Tarantino L, Capasso E, Palmieri G, Guarrasi R, Bianco M, Mamone R, Savastano C, Pisano A, Vincenzi B, Sabia A, D'Agostino A, Faiola $V$, Addeo $R$ : Sorafenib plus octreotide is an effective and safe treatment in advanced hepatocellular carcinoma: multicenter phase II So.LAR. study. Cancer Chemother Pharmacol 2010, 66:837-844.

31. Pawlik TM, Reyes DK, Cosgrove D, Kamel IR, Bhagat N, Geschwind JF: Phase II trial of sorafenib combined with concurrent transarterial chemoembolization with drug-eluting beads for hepatocellular carcinoma. J Clin Oncol 2011, 29:3960-3967.

32. Wang C, Lu Y, Wang H, Gao X, Bai W, Qu J, Xu G, Zhang Z, Zeng Z, Zhou L, An L, LV J, Yang Y: Transarterial chemoembolization with/without cryotherapy is associated with improved clinical outcomes of sorafenib for the treatment of advanced hepatocellular carcinoma. Exp Therapeut Med 2012, 4:188-196.
33. Kudo M, Ueshima K, Arizumi T: Real-life clinical practice with sorafenib in advanced hepatocellular carcinoma: a single-center experience. Dig Dis 2012, 30:609-616.

34. NCT01135056. Study to compare selective internal radiation therapy (SIRT) versus sorafenib in locally advanced hepatocellular carcinoma (HCC). [http://clinicaltrials.gov/ct2/show/NCT01135056?term=sorafenib +hepatocellular+carcinoma+yttrium-90\&rank=5]

35. Wu S, Chen JJ, Kudelka A, Lu J, Zhu X: Incidence and risk of hypertension with sorafenib in patients with cancer: a systematic review and meta-analysis. Lancet Oncol 2008, 9:117-123.

doi:10.1186/1745-6215-15-474

Cite this article as: Vilgrain et al:: Radioembolisation with yttrium-90 microspheres versus sorafenib for treatment of advanced hepatocellular carcinoma (SARAH): study protocol for a randomised controlled trial. Trials 2014 15:474.

\section{Submit your next manuscript to BioMed Central and take full advantage of:}

- Convenient online submission

- Thorough peer review

- No space constraints or color figure charges

- Immediate publication on acceptance

- Inclusion in PubMed, CAS, Scopus and Google Scholar

- Research which is freely available for redistribution

Submit your manuscript at www.biomedcentral.com/submit
C BioMed Central 